Proceedings

\title{
Revisiting Colorimetric Gas Sensors: Compact, Versatile and Cost-Effective ${ }^{\dagger}$
}

\author{
Christian Driau, Olga Casals, Ismael Benito-Altamirano, Joan Daniel Prades * \\ and Cristian Fàbrega * \\ IN²UB - MIND, Department of Electricns and Biomedical Engineering, Universitat de Barcelona (UB), \\ 08028 Barcelona, Spain; christian.driau@gmail.com (C.D.); olga.casals@ub.edu (O.C.); \\ ismael.benito@ub.edu (I.B.-A.) \\ * Correspondence: dprades@ub.edu (J.D.P.); cfabrega@ub.edu (C.F.) \\ + Presented at the 4th International Conference nanoFIS 2020-Functional Integrated nano Systems, Graz, \\ Austria, 2-4 November 2020.
}

Published: 17 December 2020

\begin{abstract}
We report on an inexpensive and very selective gas sensor implemented by simply combining colorimetric indicators casted on top of acetate-based transparent tape, with a commercial microchip adapted here to measure optical reflectance. This sensor can be easily reproduced (leading to quantitatively consistent results), refreshed and reconfigured to sense different target gases replacing only the colorimetric tape. The device may either work as sensor $\left(\mathrm{CO}_{2}\right.$ and $\left.\mathrm{NH}_{3}\right)$ or dosimeter (Formaldehyde) depending on the targeted gas.
\end{abstract}

Keywords: colorimetric sensor; Formaldehyde; optical sensor

\section{Introduction}

Colorimetric methods put at our disposal a wide arsenal of compounds and reaction mechanisms to address specific gaseous molecules. These techniques offer unbeatable levels of selectivity and specificity towards the target species. Regarding their readout, spectrophotometers offer a detailed view of the changes developed in the light absorption spectra of these indicators, but they are bulky and practicable only for research purposes or high-end applications. To achieve a continuous readout and compact form factors, development must be focused on miniaturized systems that measure color changes at specific spectral ranges [1,2]. Even though many different configurations have been proposed, most are based on confronting a narrow-spectrum light source with a photodetector. To maximize their sensitivity to small color changes, enlarging the optical path from the emitter to the detector through the indicator medium is the preferred option [3-5]. Consequently, the integration of the different components, especially concerning their optical alignment, is challenging. Additionally, the efforts in miniaturization complicate refreshing the indicator substances, which are prone to degradation over time. In summary, the continuous readout of colorimetric indicators still raises a set of inconveniences.

\section{Description of the New Method}

Here, we propose an easy-to-implement approach to colorimetric detection of gases that combines the following advantages: (1) it is based on easily accessible, off-the-shelf commercial components; (2) it is compatible with a wide range of colorimetric indicators, operating at different wavelengths; (3) it is easily resettable/refreshable with good stability in a time frame of weeks, and (4) offers an excellent repeatability among devices. Our gas sensor is based on the MAX30105 component. This integrated microsystem encloses a set of 3 internal LEDs (red, green and infrared), 
a broad band photodiode and the corresponding control, driving, acquisition and communication modules. We use this device to excite and monitor the indicator in reflection configuration.

To integrate the indicators on the readout chip, we spin coated each indicator on conventional Scotch tape. Then, the indicator-carrying pieces of tape were transferred to the readout MAX30105 chip by attaching the tape directly on the chip glass surface (Figure 1).

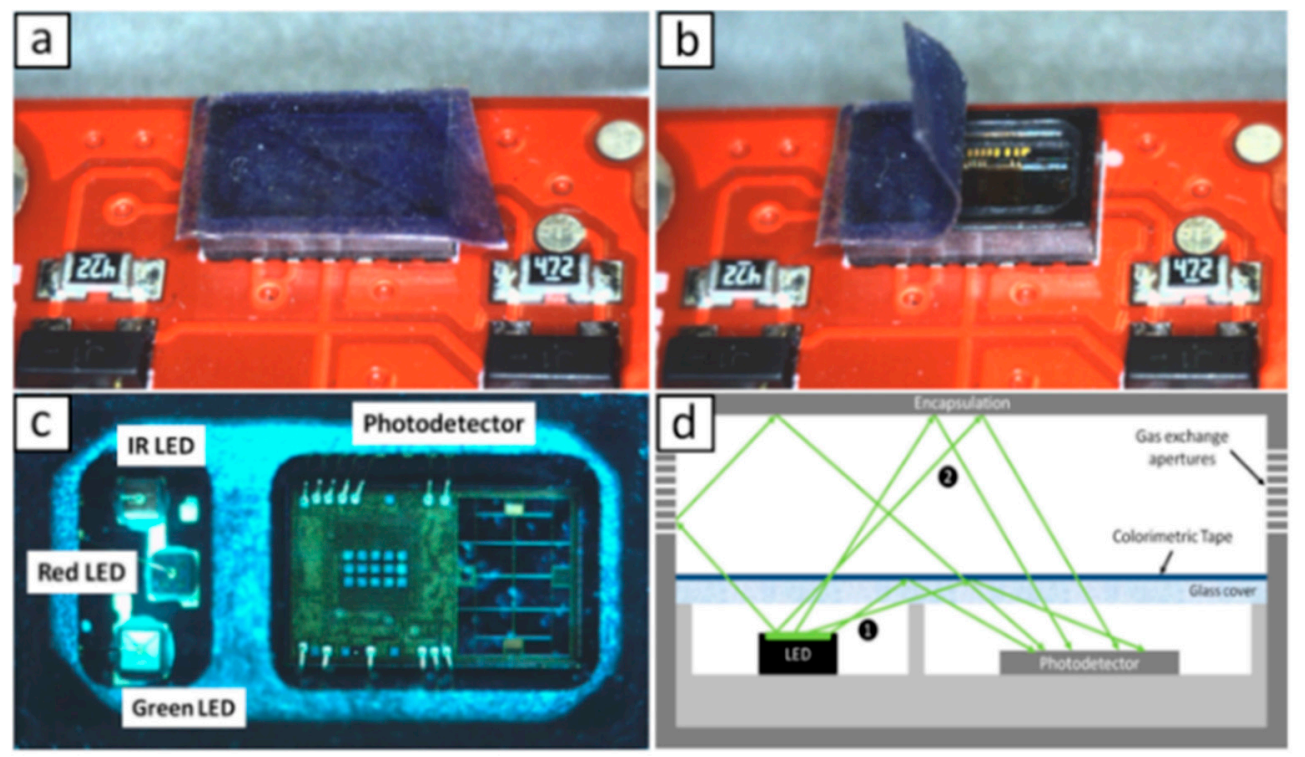

Figure 1. (a,b) Optical images of the MAX30105 component covered with a colorimetric tape. (c) A detailed image of the sensor chip with the red, green and IR LEDs and the photodetector. (d) Scheme of the proposed configuration: the photodetector will measure $\mathbf{0}$ the reflected light and 2 the transmitted light through the colorimetric tape (i.e., absorbance of the color indicator).

\section{Experimental Results}

Figure 2 shows a summary of the responses to $\mathrm{NH}_{3}$ and $\mathrm{CO}_{2}$ obtained. $\mathrm{NH}_{3}$ concentrations ranging from 2.5 to $25 \mathrm{ppm}$ were measured, with responses up to $10 \%$. The $\mathrm{CO}_{2}$ concentrations first investigated ranged from $2.5 \%$ to $50 \%$, with responses up to $12 \%$. Based on a signal-to-noise analysis, lower detection limits could be estimated in 1 and 200 ppm for $\mathrm{NH}_{3}$ and $\mathrm{CO}_{2}$, respectively. To demonstrate the selectivity of the here-proposed sensors, both devices were exposed to mixtures containing $\mathrm{NH}_{3}$ and $\mathrm{CO}_{2}$ simultaneously (Figure 2a). Clearly, each indicator responded exclusively to its target gas, without being affected by the other one (no cross-interference). Similar interference experiments were carried out with other gases (ethanol, $\mathrm{CH}_{4}$ ) leading to identical conclusions (Figure $2 \mathrm{~b}$ ). We must insist on the fact that the gas sensor behavior of our devices is completely determined by the colorimetric indicator. To illustrate this, we modified the formulation of the $\mathrm{CO}_{2}$ indicator to measure lower concentrations, which are of interest in environmental applications (25-500 ppm). Concerning repeatability, the technology also shows an exemplary comparison of the signals obtained with tow devices produced in the same batch. Despite its apparent simplicity, this approach offers very consistent and reproducible results among manually assembled devices. 

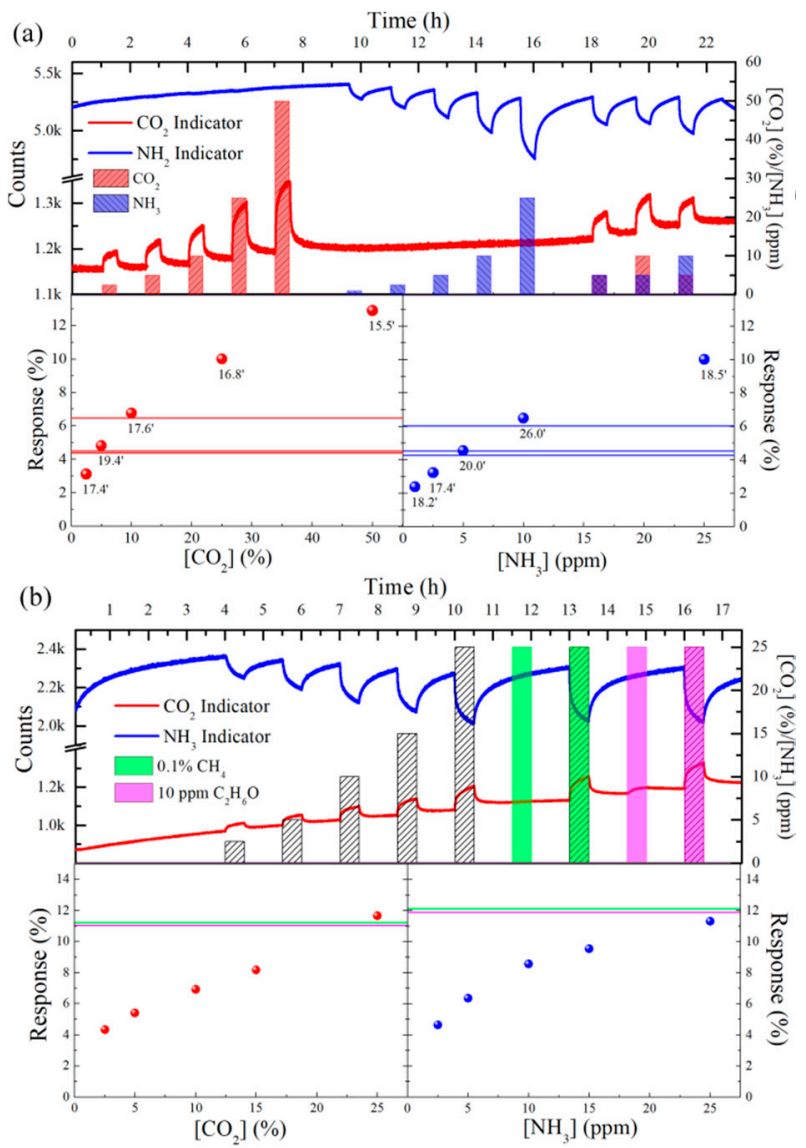

Figure 2. Dynamic response of the $\mathrm{NH}_{3}$ (blue line) and $\mathrm{CO}_{2}$ (red line) colorimetric sensors under different concentrations and cross-interference experiments (a) between them $(\mathbf{b})$ and with methane $\left(\mathrm{CH}_{4}\right)$ and ethanol $\left(\mathrm{C}_{2} \mathrm{H}_{6} \mathrm{O}\right)$. Lower panels show the response values and times for both sensors (circles and minutes) together with the responses obtained in the cross-interference experiments (horizontal lines). Data acquired with the green LED operating at $41 \mathrm{~mW}$.

Author Contributions: Conceptualization, C.F. and J.D.P.; methodology, C.F. and C.D.; software, J.D.P. and I.B.-A.; validation, C.D., and O.C.; investigation, C.D and C.F.; resources, C.F. and J.D.P.; writing-original draft preparation, C.F. and J.D.P.; writing-review and editing, C.F., O.C. and J.D.P.; visualization, I.B.-A.; supervision, C.F.; project administration, C.F.; funding acquisition, C.F. All authors have read and agreed to the published version of the manuscript.

Funding: This research was funded by Impulsa-Lavor (2018 LLAV 00013) amb el support de la Secretaria d'Universitats i Recerca del Departament d'Empresa i Coneixement. J.D. Prades acknowledge the ICREA Academia Programm.

Conflicts of Interest: The authors declare no conflict of interest.

\section{References}

1. Li, Z.; Suslick, K.S. Portable Optoelectronic Nose for Monitoring Meat Freshness. ACS Sens. 2016, 1, 13301335.

2. Lin, C.; Xian, X.; Qin, X.; Wang, D.; Tsow, F.; Forzani, E.S.; Tao, N. High Performance Colorimetric Carbon Monoxide Sensor for Continuous Personal Exposure Monitoring. ACS Sens. 2018, 3, 327-333.

3. Schmitt, K.; Rist, J.; Peter, C.; Wöllenstein, J. Low-cost fiber-optic waveguide sensor for the colorimetric detection of ammonia. Microsyst. Technol. 2011, 18, 843-848.

4. Xiong, Y.; Wang, C.-J.; Tao, T.; Duan, M.; Fang, S.-W.; Zheng, M. A miniaturized fiber-optic colorimetric sensor for nitrite determination by coupling with a microfluidic capillary waveguide. Anal. Bioanal. Chem. 2016, 408, 3413-3423. 
5. Courbat, J.; Briand, D.; Damon-Lacoste, J.; Wöllenstein, J.; De Rooij, N.F. Evaluation of pH indicator-based colorimetric films for ammonia detection using optical waveguides. Sens. Actuators B Chem. 2009, 143, 6270.

Publisher's Note: MDPI stays neutral with regard to jurisdictional claims in published maps and institutional affiliations.

(C) 2020 by the authors. Licensee MDPI, Basel, Switzerland. This article is an open access article distributed under the terms and conditions of the Creative Commons Attribution (CC BY) license (http://creativecommons.org/licenses/by/4.0/). 